\title{
Linking the Pathobiology of Hypercholesterolemia with the Neutrophil Mechanotransduction
}

\author{
Xiaoyan Zhang and Hainsworth Y. Shin \\ Additional information is available at the end of the chapter
}

http://dx.doi.org/10.5772/47867

\section{Introduction}

This chapter focuses on the potential contributions of the blood-borne neutrophils to hypercholesterolemia-related pathophysiology (e.g., thrombus formation, embolism, heart attack, stroke, etc.). Neutrophils are immersed in the cholesterol-abundant plasma of blood and play critical roles in the acute inflammatory response of the body to infection or tissue damage. Because of their high degree of sensitivity to inflammatory agonists and their arsenal of potent microbicidal and tissue degradative agents, a number of redundant cellular mechanisms exist to control or "turn-off" the inflammatory processes by these cells under physiological (non-pathological) conditions. Failure of these mechanisms leads to sustained levels of cell activity that contribute to a chronic inflammatory phenotype with the continuous release of proteases and cytokines as well as the potential to elicit non-specific damage to host tissues. Alternatively, chronic neutrophil activity may impair tissue perfusion via its effects on the rheological flow behavior of blood particularly in terms of the ability of leukocytes to transit the microcirculation[1]. Such potential damage mechanisms are thought to govern an increasing number of human pathological scenarios (e.g., Alzheimer's, diabetes, vascular disease) that correlate with a chronic inflammatory state. In this regard, chronic inflammation has gained recognition in the scientific community and even in the mainstream national media (e.g., Time[2] and Newsweek[3] magazines) as a common denominator for human diseases. The question is whether the dysregulation of neutrophil activity is a significant component of this potential disease mechanism.

We address this issue from a mechanobiological perspective by presenting evidence that supports a role of impaired neutrophil mechanotransduction of hemodynamics-derived fluid flow in the pathogenesis of hypercholesterolemia-linked diseases. For this purpose, we 
will first discuss the link between chronic inflammation and hypercholesterolemia and then highlight the neutrophil involvement in the pathophysiology of related cardiovascular diseases, e.g. atherosclerosis and microvascular dysfunction. To further exemplify this link, we will explain the potential mechanism(s) by which cholesterol in blood may impact the biochemical regulation of neutrophil activity at the cellular level. Finally, we will introduce our own evidence as well as those of others pointing to dysregulated neutrophil mechanotransduction as an important component of hypercholesterolemic pathologies.

\section{Hypercholesterolemia and chronic inflammation}

Hypercholesterolemia is the dominant risk factor for atherosclerosis and its downstream complications (e.g., heart attack, stroke, etc.). Over the past two decades, a wealth of insight has pointed to the development of atherosclerotic lesions in the large vessels (e.g., aorta, carotid, femoral artery, etc.) as occurring at the interface between hypercholesterolemia and inflammation (see reviews[4,5]). According to the current paradigm, at atheroma-prone sites, inflamed endothelial cells (due to damage or dysregulation) initiate the invasion of blood leukocytes (predominantly, monocytes) and smooth muscle cells (SMCs) into the subendothelial (e.g., the intimal) layer of the vascular wall contributing to atherosclerotic tissue remodeling, thrombosis, and finally embolus formation. The main lipid species that appear to dominate this inflammatory process are modified low-density lipoprotein (LDL) particles, particularly oxidized LDL (oxLDL), which act as potent proatherogenic and proinflammatory factors responsible for not only loading monocyte-derived macrophages with cholesterol but also directly stimulating leukocytes and other vascular wall cells (for a more complete explanation, see reviews[5, 6]).

Hypercholesterolemia also induces chronic inflammation in the microcirculation[7]. Phenotypic changes in the microvasculature are observed long before the appearance of fatty streak lesions in the large arteries of animals placed on high fat (HFD), i.e. proatherogenic, $\operatorname{diet}[8,9]$. The inflammatory phenotype of the microvessels in hypercholesterolemic animals results in increased basal levels of rolling, adherent, and emigrating leukocytes in the postcapillary venules, predominantly neutrophils, as well as enhanced production of reactive oxygen species (ROS). Hypercholesterolemia also exaggerates microvascular responses to a range of proinflammatory stimuli. For example, the postcapillary venules of LDL receptor deficient (LDLr-/-) mice, a murine model of modest hypercholesterolemia (with 3-fold higher levels of plasma cholesterol compared to their wild-type (WT) counterparts), exhibit enhanced leukocyte adhesion and albumin leakage in response to experimental ischemia/reperfusion injury as compared to those of WT mice[10]. Interestingly, similar phenotypic changes can be observed in the microvasculature of normocholesterolemic animals administered oxLDL[11, 12], suggesting that oxLDL participates in hypercholesterolemia-related microvascular dysfunction.

Although the mechanisms responsible for the induction of inflammation by hypercholesterolemia in both microvessels and larger arteries remain unclear, it appears 
that they both begin with endothelial dysfunction characterized by reduced vasodilation, a proinflammatory state, and enhanced permeability to macromolecules (e.g., lipids). However, the original triggers for this endothelial dysfunction are still controversial. In this regard, oxLDL, based on its potent proinflammatory effects, has been considered as a candidate that initiates the inflammatory responses. In fact, production and release of ROS and myeloperoxidase (MPO)[13], which play critical roles in the oxidation of LDLcholesterol conjugates and are tightly controlled under the physiological non-inflamed conditions, increase in response to hypercholesterolemia. The cellular basis for the causality between oxLDL formation and chronic inflammation, however, remains elusive.

Interestingly, the preferential formation of atherosclerotic lesions at bifurcations, severe curvatures, and stenoses in the arterial circulation strongly suggests that the hemodynamic flow environment is an important determinant in atherogenesis. Fluid flow-derived frictional (i.e., shear) stresses imposed on the surfaces of endothelium lining the vascular wall have been shown to serve an atheroprotective function when blood flow is laminar (i.e., smooth and ordered; for a more comprehensive discussion, see review[14]). For example, laminar fluid flow stimulates endothelial production of nitric oxide (NO), with vasodilatory and anti-inflammatory actions[15]. In contrast, oscillatory shear stresses enhance production and release of ROS[16]. In addition, disturbed flows lead to the upregulation of adhesion molecules on the endothelial surface (e.g., intercellular adhesion molecule-1 or ICAM-1) responsible for recruiting leukocytes to the vascular wall[17]. In effect, generation of complex distributions of fluid shear stresses on the vascular wall, such as at sites of bifurcations and branch points, appears to shift the endothelial phenotype from atheroprotective to proatherogenic.

However, complex flow fields are not sufficient for the onset/progression of hypercholesterolemia-related atherosclerosis since, for example, we are born with bifurcations and curvatures but do not develop atherosclerosis from birth. It is, therefore, clear that cardiovascular pathobiology due to hypercholesterolemia occurs at the intersection between vascular cell biology and the surrounding fluid stress environment. In this regard, it may be the sensitivity (i.e., responsiveness) of vascular cells to fluid shear stress that is altered in the face of hypercholesterolemia leading to a proinflammatory and a proatherogenic phenotype. Moreover, the endothelial cells are not the only cells in the vasculature. Neutrophils also exist in the cholesterol-enriched, fluid flow environment of the circulation and are critical for initiating acute inflammation. Recently, a growing body of evidence supporting the involvement of neutrophils has emerged.

\section{The neutrophil involvement in hypercholesterolemia-related vascular dysfunction}

Neutrophils make up the majority of the nucleated leukocytes in human blood with the remaining being monocytes and lymphocytes. As the principal gatekeepers of the acute inflammatory response of the body's immune system, neutrophils are extremely sensitive to 
inflammatory stimuli allowing them to rapidly (i.e., on the order of milliseconds) transition from an inactivated to an activated state. Upon activation, the upregulation of cell-cell adhesion molecules (e.g., selectins, integrins) enables neutrophils to roll and adhere onto the endothelium prior to their transmigration (via diapedesis) across the vascular wall and to the target tissues (i.e., sites of infection and tissue injury) where they release a potent array of biochemicals including proinflammatory mediators, ROS, and proteases to fight infection and orchestrate tissue repair. During this process, neutrophils also undergo changes in their physical attributes such as their size, deformability, and adhesiveness. It is these features that cause neutrophils to be major players in the pathobiology of hypercholesterolemiarelated cardiovascular diseases for both the macro- and micro- circulations.

\subsection{Potential roles of neutrophils in atherosclerosis}

While it has long been appreciated that monocytes and their descendants, along with $\mathrm{T}$ lymphocytes, mast cells, and platelets, contribute to the development and destabilization of atherosclerotic lesions, only recently has the neutrophil been seriously considered as a contributing factor for disease onset and/or progression. Direct evidence comes from the identification of neutrophils in different locations of atherosclerotic lesions present in hypercholesterolemic mice and humans using antibodies to neutrophil-specific antigens including Ly6G, CD177, and CD66b[18-21]. Neutrophils, in fact, have been reported to accumulate in atheroprone arteries preceding plaque formation in hypercholesterolemic murine models of atherosclerosis[18, 20]. Further evidence of a neutrophilic component in the early stages of atherosclerosis is the positive correlation between the number of circulating neutrophils and lesion sizes[18]. Experimental data also point to neutrophil infiltration into the highly inflamed areas of atherosclerotic arteries during late disease stages[19] with contribution to lesion destabilization and thromboembolus formation[21, 22].

One way chronically activated neutrophils may enter atheroprone regions in the macrocirculation are at sites of disturbed flow and recirculation[23] where their enhanced residence times promote capture at the vascular wall[24]. Alternatively, activated neutrophils may disrupt vascular (i.e., adventitial or medial) wall perfusion in the vasa vasorum (or microcirculation) of large vessels (e.g., aorta) leading to vessel tissue injury followed by atherogenesis (from within the vessel wall to the luminal surface)[25-27]. In these ways, neutrophils may initiate or exacerbate atherosclerosis at different stages via their capability to release vast amounts of ROS and proteins stored in their cytosolic granules[28]. For example, while MPO released by activated neutrophils can reduce the bioavailability of $\mathrm{NO}[29,30]$ and contribute to the onset of endothelial dysfunction, a number of granule proteins, such as LL-37, azurocidin, cathepsin G, and $\alpha$-defensins, exert direct chemotactic activity for monocytes[31, 32]. Moreover, neutrophil secretory products, e.g., $\alpha$-defensins, also promote macrophage maturation and activation, contributing to the uptake of oxLDL and the formation of foam cells[28]. Finally, neutrophil-derived proteolytic enzymes, particularly matrix metalloproteinase (MMP) -2 and $-9[28]$, play critical roles in plaque destabilization and eventual rupture. 


\subsection{Effects of neutrophils on microvascular dysfunction}

Similar to situations in large arteries, activated neutrophils promote microvascular dysfunction through the sustained release of proinflammatory, cytotoxic, and degradative agents. However, while leukocytes have no significant effect on the macrovascular flow properties of blood (which is dominated by the substantially greater numbers of erythrocytes), these cells, particularly the neutrophils, influence blood flow in the microcirculation (Figure 1). This is because vessel diameters in the microcirculation are in the range of $6-100 \mu \mathrm{m}$, which are comparable in size to the diameters of leukocytes.

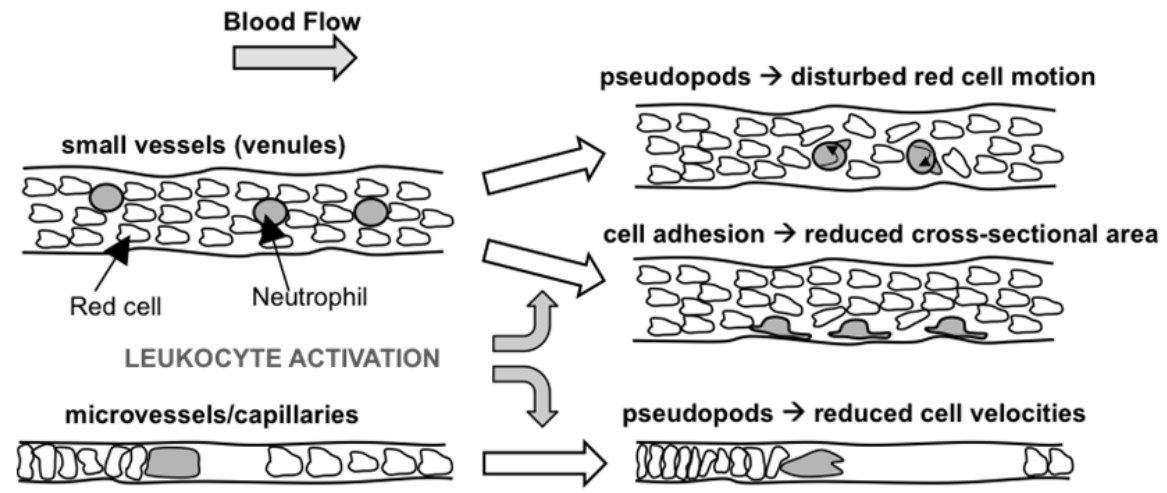

Figure 1. Potential rheological effects of leukocyte activation on the blood flow in the microcirculation. Sustained activation, e.g., due to proinflammatory stimuli, hinders leukocyte passage through the small vessels either by promoting pseudopod projections or through enabling cell adhesion to the vascular wall. Ultimately, these may elevate peripheral resistance and contribute to microvascular dysfunctions (adapted from Shin, H.Y., et al., 2011[79]).

The relatively comparable size scales of the neutrophil and vessel diameters are important to note since the quiescent neutrophil under physiological (i.e., non-inflamed) conditions is capable of efficient transit through the microvessels due to their inherently round, deformable, and non-adhesive state. On the other hand, cell activation physically hinders the passage of neutrophils through the small vessels of the microcirculation[1]. Pseudopods projected by activated neutrophils, while enabling cells to attach to other cells (e.g., endothelial cells, other blood cells) or phagocytose particles, also contribute to a reduction in cell deformability due to their enriched content of F-actin, and increases in geometric size and irregularity[1,33], all of which serve to increase leukocyte transit time or enhance leukocyte retention in the microvasculature[34, 35]. In turn, activated leukocytes disrupt the motion of erythrocytes, leading to increases in the apparent viscosity of blood and microvascular resistance[1, 34, 36, 37]. Moreover, activated neutrophils are hyperadhesive and exhibit extensive interactions with other leukocytes or platelets, e.g. during hypercholesterolemia[7], which may also enhance the apparent viscosity of blood. Finally, once neutrophils adhere to endothelium, they further increase flow resistance by reducing microvessel diameters (resistance $\propto 1 /[\text { diameter] }]^{4}$ [34, 37]. The 
state of neutrophil activation is, thus, a critical determinant of tissue blood flow and perfusion.

In summary, as a result of their arsenal of noxious agents and their effects on microvascular blood flow, it is evident that tight regulation of neutrophil activity is an essential requirement for a healthy circulation. A failure to either prevent or "turn-off" cell activity, e.g., due to hypercholesterolemia, leads to sustained neutrophil activation which has potential impacts not only in terms of the initiation and progression of atherosclerosis in large arteries but also as it relates to microvascular blood flow and downstream tissue perfusion.

\section{The influence of cholesterol on neutrophil activity}

One way hypercholesterolemia may influence the activation state of neutrophils is to modify the lipid composition of biological membranes. Cholesterol is an essential component of mammalian cell membranes. Approximately $90 \%$ of the free (i.e. unesterified) cholesterol in cells resides in the plasma membrane[38]. These sterol molecules not only maintain the integrity of cell membranes, but also play an important role in regulating membrane properties (e.g., microviscosity) and functions (e.g., via their influence on membrane-bound signaling components). In addition to de novo biosynthesis, mammalian cells can take up cholesterol from the extracellular milieu. Exposure to elevated cholesterol levels both in vivo and in vitro enhances cholesterol abundance within the plasma membrane of neutrophils and other blood cells[39-42]. These findings, in conjunction with the wealth of evidence demonstrating the influence of the extracellular cholesterol levels on neutrophil activity, point to membrane cholesterol enrichment as a potential link between hypercholesterolemia and chronic neutrophil activity. To better understand this link, we next describe the possible cholesterol uptake pathways, the influence of cholesterol on physicochemical properties of the cell membrane, and lastly, the influence of cholesterol on neutrophil activity.

\subsection{Transport of extracellular cholesterol into the plasma membrane}

Due to its insolubility in aqueous media, cholesterol must be transported complexed to carrier molecules, i.e. within the hydrophobic cores of lipoproteins[43]. Lipoproteins (e.g., LDL) in the blood plasma are positioned in close proximity to the circulating blood cells. Conditions that elevate cholesterol-enriched lipoprotein levels may thus favor cholesterol transport into the membranes of these blood cells[39, 42]. In the laboratory, cyclodextrin derivatives (e.g., methyl- $\beta$-cyclodextrin or $M \beta C D$ ), synthetic cholesterol carrier molecules, are commonly used to alter membrane cholesterol abundance. Such treatments elicit acute changes in membrane cholesterol levels and downstream cell activity indicating the existence of mechanism(s) that permit rapid transport of cholesterol into nearby cell membranes. Cholesterol uptake may occur by either receptor dependent/independent endocytosis followed by rapid membrane mobilization[44] or direct exchange between the hydrophobic environments of carrier molecules and the lipid bilayer(Figure 2). 


\subsubsection{Receptor-dependent endocytosis}

For a variety of cell types (including leukocytes)[45], LDL-cholesterol is taken up in vivo mainly through LDL receptor (LDLr)-mediated endocytosis. LDLr expression is subject to feedback regulation and, thus, is unlikely to contribute significantly to the overaccumulation of cellular cholesterol[43]. Cellular uptake of cholesterol can also occur via endocytosis mediated by other receptors[43]. Potentially, these pathways can load cholesterol continuously into the cell leading to cholesterol elevations in the plasma membrane[43]. For example, a family of scavenger receptors have been identified on monocytes, macrophages, and SMCs[6] that, by binding to modified LDL (e.g., oxidized and acetylated LDL) with high affinity, account for the majority of cholesterol uptake by these cells[6]. To our knowledge, such scavenger receptors have not yet been identified for neutrophils.

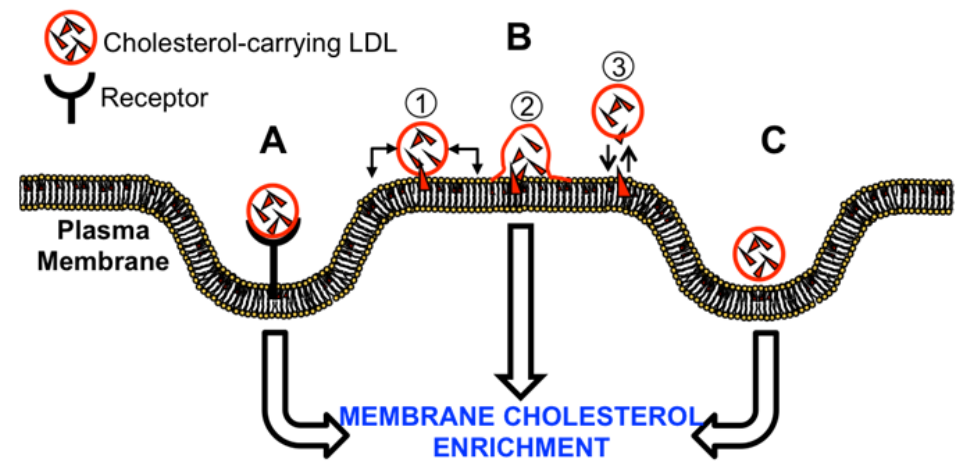

Figure 2. Schematic representation of three possible modes of cholesterol uptake. A: Receptormediated endocytosis; B: Direct surface exchange of cholesterol between extracellular carrier molecules and plasma membrane which may occur due to the formation of a transient collision complex without (1) or with (2) membrane fusion or resulting from diffusion across the aqueous phase (3); C: Receptor-independent endocytosis.

\subsubsection{Receptor-independent endocytosis}

The entire LDL particle can be internalized as a result of fluid or bulk endocytosis without receptor-mediated LDL binding to the cell surface[46]. It is taken up at a rate strictly proportional to its concentration in the extracellular milieu[43]. Alternatively, some LDL, e.g., cationized LDL, can also be taken up by the cell through a non-specific low affinity adsorptive endocytotic process. In this case, endocytosis occurs after cationized LDL binds to the negatively charged membrane surface[47]. For both of these modes of endocytosis, cholesterol transport is not influenced by intracellular cholesterol levels and thus may lead to progressive cholesterol uptake[43].

\subsubsection{Cholesterol surface exchange}

Cholesterol may also directly enter or exit the plasma membrane[48]. In this case, free cholesterol is exchanged between the hydrophobic cores of the plasma membrane and 
extracellular carriers (e.g., lipoproteins). The direction of net flux of cholesterol is governed by its concentration gradient between the lipid bilayer and the carrier molecules. Two mechanisms for this surface transfer have been proposed: 1) formation of transient collision complexes with/without membrane fusion and 2) direct diffusion across the aqueous phase. In principle, these transport modes follow similar kinetics with transfer rates depending on the concentrations and structures of both donor (e.g., extracellular LDL) and acceptor (e.g., cells) particles (for more details, see review[48]). This level of complexity contributes, in part, to the diverse half-times ranging from seconds to hours measured for the uptake of cholesterol by human erythrocytes[49]. Finally, this pathway is not under feedback control.

\subsection{The influence of cholesterol on the plasma membrane dynamics}

In the lipid bilayer, cholesterol orients with its polar hydroxyl group encountering the aqueous phase and the hydrophobic steroid ring parallel to and buried in the hydrocarbon chains of the phospholipids[50]. This unique orientation allows cholesterol to interact with membrane phospholipids and sphingolipids and thus influence their physicochemistry. Along these lines, cholesterol influences the physical and biological properties of the lipid bilayer and, in doing so, impacts the functions of membrane signaling molecules.

\subsubsection{Effects of cholesterol on the physical properties of cellular membranes}

A key function of cholesterol is to modulate the fluidity (i.e., the inverse of microviscosity) of the lipid bilayer. The close inter-positioning of sterols (i.e., cholesterol) between neighboring membrane phospholipids imposes a degree of immobility on the carbon atoms nearest the membrane surface, while increasing the freedom of motion deep within the hydrophobic core of the membrane[51]. In this regard, membrane fluidity reflects the temperature-dependent influence of cholesterol on the gel to liquid-crystal (i.e., solid-like to fluid-like) phase transition of the lipid bilayer[51]. Under physiological conditions (i.e., $\left.37^{\circ} \mathrm{C}\right)$, biological membranes adopt a liquid crystalline state whereby increases and decreases in cholesterol content reduce and enhance membrane fluidity, respectively[42, $52,53]$.

Operationally, membrane fluidity refers to the ensemble of physical properties that govern the motion of the phospholipid molecules in a membrane, including segmental, rotational, lateral, and translational motions[54]. In this fashion, lipid bilayer fluidity can physically influence the dynamics of membrane-associated molecules including proteins that drive downstream cell functions. Two mechanisms have been proposed to explain how membrane fluidity alters the activities and functions of membrane proteins. One mechanism occurs through effects on protein mobility, particularly lateral diffusion which impacts collisional encounters[54]. This effect appears to be of physiological significance particularly for diffusion-controlled processes that are mediated by large membrane proteins. For example, modulation of membrane fluidity influences Ca++-dependent cAMP signaling through changes in protein mobility that governs the coupling between hormone receptors and the adenylate cyclase catalytic unit $[55,56]$. Alternatively, membrane fluidity can also 
influence the structural flexibility of membrane proteins and, thus, their ability to adopt an optimal conformation for activity[57]. Membrane fluidity has, in fact, been reported to impact the conformation-dependent activation of G-protein coupled receptors (GPCRs) on endothelial cells by affecting changes in the protein tertiary structure[58].

\subsubsection{Role of cholesterol in lipid raft structure and function}

Lipid rafts are nano-scale microdomains that are abundant in the plasma membrane. Structurally, lipid rafts consist of dynamic assemblies of cholesterol and sphingolipids in the outer leaflet of the phospholipid bilayer[59]. The preponderance of saturated hydrocarbon chains in raft sphingolipids renders lipid rafts with a distinct liquid-ordered (i.e., solid-like) phase that is dispersed in the liquid-disordered matrix of the lipid bilayer[60]. One important property of lipid rafts is that they include or exclude proteins to variable extents depending on the raft affinity of proteins[59]. Once individual rafts cluster, they spatially facilitate interactions between raft proteins and expose them to a new membrane environment that is enriched in accessory enzymes and/or second-messenger molecules. In doing so, lipid rafts serve to efficiently initiate and/or amplify signaling cascades. As such, lipid rafts act as signaling platforms that orchestrate outside-in and inside-out signal transduction. Interestingly, these cholesterol-rich microdomains have also been implicated as mechanotransduction centers such as caveolae, a subtype of lipid rafts that reportedly play a role in endothelial mechanotransduction of shear stress and pressure[61-63].

Cholesterol is required to support the formation of lipid rafts and maintain their functionality. It condenses the packing of sphingolipids in the exoplasmic leaflet by occupying the spaces between their saturated hydrocarbon chains near the hydrophilic polar head groups. In this way, cholesterol content and organization influence the stability of lipid rafts with an impact on their capacity to interact with target proteins. Removal or depletion of cholesterol from the plasma membrane using $\mathrm{M} \beta \mathrm{CD}$ has been widely used to disrupt rafts and disperse raft proteins into the liquid-disordered matrix of the cell membrane[59]. Treatment of cells with cholesterol-sequestering agents (e.g., filipin or nystatin) or inhibition of cholesterol biosynthesis (e.g., lovastatin) as well as addition of exogenous cholesterol into cell membranes also disrupts raft structure leading to an impact on the functions of raft proteins[59]. As a consequence of these lipid raft-related perturbations, neutrophil functions (e.g., chemokine-induced calcium signaling, extracellular regulated kinase activity, cell polarization, shape change, adhesion, migration, integrin expression, and actin polymerization) are altered[64-68].

\subsection{Effects of elevated cholesterol environments on neutrophil activity}

Up to this point, we have described how perturbations in extracellular cholesterol levels modify the membrane physicochemistry and the mode by which these modifications may influence membrane protein-related signaling in the neutrophil. The altered cell signaling capacity of membrane-bound proteins is followed by changes in cell behavior that contribute to the principal role of the neutrophil as the first responder to tissue damage and 
infection. In this way, the influence of membrane cholesterol on the ability of the neutrophil to sense its environment extends to basic cell functions including cell adhesion and migration, phagocytosis, ROS production, and degranulation. We will discuss the effects of cholesterol on these cell functions in order to illustrate the link between the lipid bilayer properties and the control of neutrophil activation.

\subsubsection{Expression of membrane adhesion molecules}

Upon agonist stimulation, neutrophils exhibit upregulated expression of adhesion molecules that facilitate their recruitment to sites of inflammation by enabling their binding to other cells (e.g., leukocytes, platelets, endothelium)[33]. Two classes of adhesion molecules govern leukocyte interactions with other cells: selectins and integrins. In addition to the ligands for platelet $(\mathrm{P})$ - or endothelial (E)- selectins, neutrophils constitutively express leukocyte (L)-selectins and $\beta_{2}$ (i.e., CD18) integrins, which participate in their initial capture and firm adhesion to other cells, respectively[33]. Currently, the impact of hypercholesterolemia on expression of the selectin family of adhesion molecules is unclear since neutrophils in a cholesterol-rich environment have been reported to exhibit both elevated surface expression[69] and cleavage of L-selectins[70]. In the case of the integrins, surface levels of CD18, particularly Mac-1 (CD11b/CD18), are elevated on neutrophils exposed to a hypercholesterolemic environment both in vitro and in vivo[29, 69, 70]. Notably, surface expression of Mac-1 by neutrophils in hypercholesterolemic patients positively correlates with serum cholesterol levels[29]. But, cholesterol enrichment does not appear to alter the expression of LFA-1 (CD11a/CD18)[69], another CD18 subtype. Thus, the influence of extracellular cholesterol levels on neutrophil adhesion molecule expression is receptor-specific.

Moreover, neutrophils exposed to elevated cholesterol levels undergo increased adhesive interactions with other cells. For example, neutrophils with increased membrane cholesterol exhibit enhanced tethering and firm arrest on activated endothelial cell monolayers[41, 71, 72]. Moreover, neutrophils exposed to hypercholesterolemia display increased heterotypic adhesion to platelets[73] as well as increased homotypic aggregation in response to $10 \mu \mathrm{M}$ $\mathrm{N}$-formyl-Met-Leu-Phe (fMLP)[74]. These studies confirm that cholesterol-dependent modulation of adhesion molecule expression has an impact on neutrophil adhesion to other leukocytes, platelets, or the endothelium lining the blood vessel lumen.

\subsubsection{ROS production}

Neutrophil-derived ROS includes superoxide $\left(\mathrm{O}^{2-}\right)$, hydrogen peroxide $\left(\mathrm{H}_{2} \mathrm{O}_{2}\right)$, hydroxyl radicals $(-\mathrm{OH})$, and NO-related oxidants. Notably, total production of ROS by neutrophils in a hyperlipidemic environment positively correlates with levels of triglycerides and LDL but not with total amount of cholesterol in the plasma[75]. Recent studies, however, did demonstrate a positive correlation between $\mathrm{O}^{2-}$ release rate and plasma cholesterol levels[29]. Interestingly, enhanced $\mathrm{O}^{2-}$ release by neutrophils was detected in other clinical states associated with cardiovascular complications, namely hypertension and diabetes[76, 
77], which are usually accompanied by hyperlipidemia. In fact, elevations in extracellular cholesterol levels have been shown to enhance neutrophil respiratory burst in response to agonist stimulation. Moreover, plasma activity of superoxide dismutase (SOD), which scavenges ROS, decreases with increases in total cholesterol[75].

\subsubsection{Degranulation}

Neutrophils contain four main types of granules: primary, secondary, and tertiary granules as well as secretory vesicles. These granules contain a multitude of cytokines (e.g., interleukins, tumor necrosis factor- $\alpha$, etc.), enzymes (e.g., MPO, etc.), and proteases (e.g., cathepsins, MMPs, etc.). Upon activation, neutrophils degranulate and release these bioactive mediators into the extracellular milieu. Interestingly, although neutrophils from hyperlipidemic patients contain significantly lower levels of intracellular MPO, sera from these patients exhibit significantly higher levels of $\mathrm{MPO}$ [29]. These results point to a degranulation process that further links the activation state of neutrophils with the cholesterol levels in the blood environment.

\section{Membrane cholesterol and the neutrophil mechanosensitivity to shear stress}

In addition to the presence of inflammatory stimuli (e.g., oxLDL), elevated neutrophil activity in hypercholesterolemia may result from defects in their mechanotransduction of fluid shear stress, a control mechanism to prevent spontaneous neutrophil activity under physiological conditions[78, 79]. In this regard, the mechanosensitivity of neutrophils may serve as a key regulator of the inflammatory status of the circulation. We will first define the leukocyte mechanosensitivity to shear followed by a brief discussion of cellular mechanisms that link the extracellular flow environment to downstream neutrophil functions. Interestingly, such mechanotransduction processes occur across the plasma membrane that plays a critical role in regulating the activity of membrane proteins as well as the transmembrane movement of bioactive molecules. The direct contact of cell membrane with the extracellular flow environment makes it a likely target of local environmental factors (e.g., enhanced cholesterol abundance) that influence the neutrophil responsiveness to mechanical stimuli.

\subsection{Regulation of neutrophil activity by fluid flow-derived shear stress}

Neutrophils, either freely suspended in the bloodstream or adhered to/migrating on vascular endothelium, sense and respond to fluid shear stress[80-82]. Fluid shear stress (ranging from approximately 1 to $10 \mathrm{dyn} / \mathrm{cm}^{2}$ ) minimizes neutrophil activity levels[78]. The most obvious manifestation of the cell-inactivating effects of shear exposure on cell activity is the retraction of existing pseudopodia by non-cytokine-stimulated human neutrophils adhered to a surface and subjected to a non-uniform flow field imposed by a micropipette with a tip of diameter in the range of $4-8 \mu \mathrm{m}$ [82] (Figure 3A). This situation models brief 
and spontaneous periods of blood stasis followed by reperfusion, a typical scenario in the microvessels. Under this condition, neutrophils sediment, attach, extend pseudopods, and migrate on the vascular endothelium. Upon reintroduction of fluid flow, these cells retract pseudopods and detach into the flow field in a mechanobiological fashion. Such a scenario has been documented using intravital microscopy of microvascular networks of rodents (e.g., mesentery, spinotrapezius muscle, cremaster muscle)[81-83]. The ability of shear stress to minimize pseudopod activity has been further confirmed for non-adherent heterogeneous leukocyte populations[84] exposed to a constant shear field (5 dyn/ $\left.\mathrm{cm}^{2}\right)$ in a cone-plate viscometer (Figure 3B).
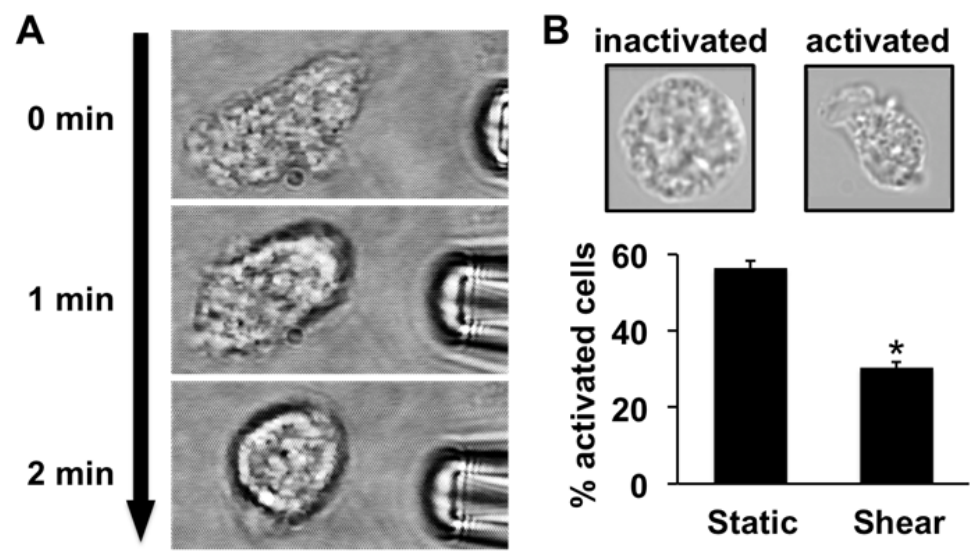

Figure 3. Deactivation of neutrophils under flow stimulation. A: A migrating/adherent neutrophil exposed to a micropipette flow $\left(\sim 2 \mathrm{dyn} / \mathrm{cm}^{2}\right)$ for $2 \mathrm{~min}$. B: Non-adherent neutrophils in suspension exposed to cone-plate shear $\left(5 \mathrm{dyn} / \mathrm{cm}^{2}\right)$ for $10 \mathrm{~min}$. Bars are mean percentage of activated cells with pseudopods (see image insets) in each population tested $\pm \mathrm{SEM} ;{ }^{*} \mathrm{p}<0.05$ compared to static condition using paired Student's t-test.

Notably, impairment of shear-induced pseudopod retraction by treating neutrophils with cell agonists above threshold concentrations, e.g. fMLP $\left(>10^{-8} \mathrm{M}\right)$, commits these cells to an activated (inflamed) phenotype and leads to their microvascular entrapment due to increases in adhesivity, size, and stiffness[35, 81, 82]. Thus, during inflammation, the biochemical milieu of the neutrophil overrides mechanobiological deactivation. Exposure to shear of magnitudes typically found in the macro- and micro- circulations is also associated with other attributes of neutrophil deactivation such as decreased surface expression of integrin receptors (i.e., CD18), depolymerization of the F-actin cytoskeleton, cell detachment, and attenuated phagocytic activity[81, 82]. Moreover, shear stress exposure enhances caspase 3-dependent apoptosis[85], in line with the relatively short lifespan (18 to $24 \mathrm{hrs}$ ) of these cells when they are passively circulating in the physiologic bloodstream. These observations support the key role of fluid flow-related shear stress as a biophysical stimulus that promotes neutrophil inactivation when cell activity is below a threshold level. As such, the mechanical influence of fluid flow serves an anti-inflammatory role. 


\subsection{Shear stress mechanotransduction at the neutrophil surface}

An understanding of the fluid flow mechanoregulation of neutrophil activity in the circulation reveals clues regarding how impaired mechanosensitivity to flow may be a mitigating factor for hypercholesterolemic disorders. Membrane detachment during pseudopod retraction by migrating neutrophils in response to fluid shear stress points to two fundamental requirements that must be fulfilled by the cell signaling apparatus: 1) depolymerization of the F-actin cytoskeleton that serves as a structural and a signaling scaffold for neutrophil motility and 2) rapid disengagement of adhesion receptors that anchor the pseudopod to the underlying substrates. For suspended neutrophils, similar events are needed but, in this case, mechanisms must be in place to prevent the expression of adhesive proteins or interfere with engagement of adhesion molecule with substrates (e.g. foreign surfaces, other cells) presenting counter-receptors. These fundamental requirements point to the neutrophil surface components as critical players in mechanotransduction since the cell must sense the extracellular flow environment and remediate its interactions with the cellular microenvironment (e.g., the surrounding matrix and cells).

\subsubsection{GPCRs and shear stress control of neutrophil pseudopod activity}

Shear stress-induced pseudopod retraction by neutrophils occurs in parallel with a rapid decrease in F-actin content[86, 87]. Typically, remodeling of the F-actin cytoskeleton in leukocytes is controlled by the Ras superfamily of small guanine triphosphate (GTP)binding proteins, particularly the small GTP-binding phosphatases (GTPases) including Rac1, Rac2, cdc42 and members of the Rho family (as reviewed in the literature[88-90]). Rather than stimulating the activity of molecules that coordinate pseudopod retraction (e.g., RhoA, MLCK), fluid shear stress appears to either inhibit (e.g., possibly through release of an inhibitor) or interfere with the ability of neutrophils to form and sustain pseudopod projections via reducing cytosolic activity of the key small GTPases (e.g., Rac1, Rac2) involved in actin polymerization[83]. These reported effects point to the actions of fluid shear stress on G protein signaling downstream of GPCRs that regulate neutrophil chemotaxis, such as the formyl peptide receptor (FPR).

Notably, fMLP, a ligand for FPR, dose-dependently impairs neutrophil pseudopod retraction responses to shear stimulation[81]. Along this line, HL-60-derived neutrophils subjected to shear stress exhibit reduced activity of Gai downstream of FPR[91]. A critical piece of evidence pointing to FPR as a mechanosensory regulator of pseudopod retraction is the observation that transfection of FPR expression plasmid in undifferentiated HL-60 cells not only confers expression of this receptor but imparts on these cells the ability to form pseudopods that retract under the influence of fluid shear stress[91]. Furthermore, HL-60 promyelocytes differentiated into neutrophils and subsequently transfected with siRNA to silence FPR expression exhibit an attenuated pseudopod retraction response to shear exposure, despite the fact that these cells retain the ability to project pseudopods because of the presence of other cytokine-related GPCRs[91]. Together, these observations point to a 
role of fluid flow in regulating the activity of membrane-associated receptors by establishing the importance of membrane-bound GPCRs, specifically FPR, in the neutrophil pseudopod retraction response to shear stress. In conjunction with the dependence of GPCR activity on the membrane cholesterol content, it is conceivable that the influence of shear stress on GPCR activity is impacted by perturbations in extracellular cholesterol abundance and their effects on the cell membrane properties.

\subsubsection{Cell surface CD18 integrins and shear stress regulation of neutrophil adhesion}

Pseudopod retraction by migrating neutrophils subjected to fluid flow depends on their expression levels of CD18 integrins[92], consistent with the requirement of these receptors for cyclical pseudopod projection and retraction[93]. In addition to modulating CD18 interactions with their ligands (e.g. ICAM-1) during inflammation[94, 95], fluid shear stress appears to regulate integrin dynamics on the neutrophil surface under conditions that mimic low activation states by redistributing these receptors from areas of maximal shear stress to regions where shear is minimal, i.e. at focal adhesions. Moreover, shear exposure reduces CD18 levels on the surfaces of migrating, and also non-adherent, neutrophils even in the presence of inflammatory mediators, e.g. fMLP[81, 96]. Considering the role of CD18 in strengthening neutrophil attachment to the vascular wall, shear-mediated reductions in CD18 likely diminish the ability of cells to maintain adhesive attachments[97]. In this way, shear-mediated reductions in CD18 serve an anti-inflammatory role that ensures neutrophils in a non-inflamed environment remain in a non-adhesive state.

The mechanism underlying shear-induced reductions in CD18 surface levels involves proteolysis that occurs on the surfaces of migrating and suspended neutrophils. Proteolysis modulates the levels of a wide variety of transmembrane receptors on the neutrophil surface including L-selectin (involved in rolling interactions with endothelium)[98] and CD43, an anti-adhesive mucin-like molecule[99]. CD18 integrins also undergo cleavage of the intracellular domain by calpain to promote detachment of the cell uropod during neutrophil migration[100]. But shear-induced truncation of CD18 integrins differs from calpainmediated cleavage in that the former involves lysosomal cysteine proteases (e.g., cathepsin B) that exert extracellular activity[96, 97]. Notably, the cell membrane is critically positioned between the intracellular levels, and the extracellular actions, of these proteases.

Additionally, cleavage of CD18 integrins under fluid flow also requires conformational changes in their extracellular domains[96]. Conformational activity of CD18 integrins involves shifts in the protein tertiary structure from a closed-bent to an open-extended configuration[96]. In the case of cytokine stimulation, this conformational change exposes ligand binding sites[101] that promote cell capture onto the vessel wall[95, 102]. Another consequence of CD18 conformational changes, which occur upon shear stress exposure, is to expose proteolytic cleavage sites[96]. With this evidence in mind, it is apparent that the physicochemical state of the cell membrane is a key factor in neutrophil mechanosensitivity that directly or indirectly affects the ability of shear stress to unfold the CD18 ectodomain. 


\subsubsection{RNS and ROS in shear mechanotransduction}

Reactive nitrogen species (RNS; e.g., NO) and ROS are multi-functional free radical mediators of acute inflammation serving not only as anti-microbial agents but also as biological second messengers that influence leukocyte functions (e.g., chemotaxis, phagocytosis, etc.)[103, 104]. NO from exogenous and endogenous sources (such as membrane-associated NO synthase) inhibits neutrophil recruitment out of the microvasculature during acute inflammation[105, 106]. Interestingly, NO also enhances neutrophil pseudopod retraction in response to shear stress and counteracts the blocking effects of cell agonists (e.g. fMLP and platelet-activating factor)[81]. In contrast, ROS, particularly $\mathrm{O}^{2-}$, interferes with the neutrophil shear response and is thought to contribute to the blocking effects of cell agonist, e.g. fMLP, on flow-induced pseudopod retraction[84].

Notably, the fact that inhibition of NO synthase activity in neutrophils has no effect on shear-induced pseudopod retraction[81] points to an exogenous source and an extracellular role for NO. This finding leaves open the possibility that the facultative effects of NO on the neutrophil shear response (i.e., pseudopod retraction) result from its ability to scavenge $\mathrm{O}^{2-}$ [103] and, in this way, mediate cell pseudopod activity[107, 108]. In support of this, SOD (an $\mathrm{O}^{2-}$ scavenger) also enhances the shear responses of fMLP-stimulated neutrophils[84]. Thus, $\mathrm{O}^{2-}$ is a critical mediator for neutrophil shear response. Since the cell membrane, particularly cholesterol-enriched lipid rafts, plays an important role in regulating the production/release of $\mathrm{O}^{2-[109]}$, its state may indirectly influence neutrophil mechanosensitivity to shear stress.

\subsection{Neutrophil mechanosensitivity and cardiovascular disease}

The accumulated evidence reported in the vascular mechanotransduction literature (see reviews[23, 78]) points to the following general paradigm. Exposure of vascular cells to physiological flows under normal (i.e., non-diseased, non-inflamed) conditions correlates with quiescence (i.e., baseline activity). This paradigm resulted from a multitude of studies that selectively examined the activity of various signaling pathways and putative force sensors in response to applied mechanical stresses. They, however, overlooked a subtle, but equally important, factor: mechanosensitivity or the degree to which cells respond to mechanical stresses. Just as biochemical perturbations (e.g. pathogens, inflammatory agonists) temporally and dose-dependently alter vascular cell activity leading to pathogenesis, so must changes in cell mechanosensitivity impact circulatory health.

Neutrophils experience wide variations in fluid stresses as they pass through the circulation and, thus function "normally" under a diverse array of mechanical stress distributions and magnitudes. In other words, aberrant mechanical stresses are unlikely to be a cause of cell dysfunction. What may change and contribute to "abnormal" behavior is their sensitivity to the surrounding fluid flow mechanoenvironment with a negative impact on the ability of fluid shear stress to deactivate the neutrophils. Along this line, the work of Geert SchmidSchönbein at the University of California, San Diego has demonstrated that attenuated neutrophil shear responses contribute to the microvascular pathobiology observed in spontaneously hypertensive rats (SHRs)[110] and, in doing so, illustrated the potential impact of impaired shear stress mechanotransduction on cardiovascular health. 


\subsubsection{Impaired fluid shear responses and downstream effects on vascular pathophysiology}

Significant features of the blood from SHRs are elevated numbers of circulating neutrophils, suppressed expression of adhesion molecules (e.g., selectins, CD18), and an activated phenotype[111-113]. Although the increased activity of neutrophils is not associated with increased adhesion to microvascular endothelium[78], their increased numbers raise peripheral vascular resistance[110]. One possible explanation is that circulating activated neutrophils in SHRs release vasoactive substances that constrict the small arteries and arterioles; this has been documented for atherosclerosis[114-116]. Extensive evidence, however, points to a hemorheological effect of leukocyte activation on microvascular resistance[1, 34, 36]. Specifically, the disturbed motion of white blood cells, due to pseudopod projection, significantly reduces erythrocyte velocities in the microcirculation increasing hemodynamic resistance and upstream blood pressures[36, 110] (see Figure 1).

The key evidence for the involvement of fluid flow mechanotransduction in microvascular abnormalities due to hypertension is that neutrophils from SHRs lack the ability to retract pseudopods in response to shear stress; in some cases, cells extend cellular projections under flow stimulation[110]. The underlying mechanism associated with the blockade and possible reversal of the pseudopod retraction response to shear stress reportedly involves the dependence of blood pressure in SHRs on the plasma level of glucocorticoid-related steroid hormones and the density of glucocorticoid receptors on the neutrophil surface[117, 118]. In line with this, glucocorticoid-treated[119] rats, like SHRs, exhibit elevated peripheral resistance in parallel with elevated numbers of neutrophils that lack a pseudopod retraction response to shear stress. Taken together, leukocyte shear mechanotransduction appears to be critical for the maintenance of a healthy circulation, particularly the microcirculation. Failure of this regulatory mechanism, e.g., due to impaired cell mechanosensitivity resulting from a pathological blood environment, may not only lead to sustained neutrophil activation but also result in disturbed blood flow. In this way, aberrant neutrophil mechanotransduction may contribute to microvascular damage that exacerbates ischemia-reperfusion injury or leads to peripheral vascular disease and downstream organ/tissue injury.

Studies on spontaneous hypertension also reveal a key point. Factors that drive phenotypic changes in neutrophils (e.g., from an inactivated to an activated state) dramatically alter their ability to sense the surrounding flow environment (i.e., mechanosensitivity) leading to the development of pathological behavior, including immune suppression. Intuitively, cell mechanosensitivity depends on the number and activity of proteins "moonlighting" as putative mechanosensors embedded in the cell membrane positioned at the interface between the intra- and extra- cellular milieu. These studies further strengthen the argument that the plasma membrane is a critical determinant of neutrophil mechanosensitivity.

\subsubsection{The plasma membrane and shear stress mechanosensitivity}

The fact that shear stress-induced neutrophil deactivation (e.g., FPR deactivation, G protein signaling, CD18 cleavage, pseudopod retraction, etc.) occurs in the absence of any passive 
cell deformation due to flow[120] substantiates the presence of a cell surface component(s) that transduces flow stimulation. Interestingly, neutrophils retract pseudopods independently of the fluid shear stress distribution imposed on the cell surface[82]. Thus, membrane properties appear to outweigh the location of mechanosensors on the cell surface. Moreover, non-adherent neutrophils respond to shear stress further emphasizing the importance of cell membrane-mediated over cell deformation-based (e.g., cytoskeletonrelated, cell adhesion-dependent) neutrophil mechanotransduction.

The membrane itself may act as a mechanotransducer either via stress-induced changes in its fluidity[121-123] or through lipid $\operatorname{rafts}[62,63,124]$. However, the concept that the membrane serves as a fluid stress sensor lacks the specificity that explains the diversity of cell type-specific responses to shear. An alternative, more plausible, viewpoint is that the cell membrane serves as mechanotransduction center for the cell. Along this line, the specificity associated with mechanotransduction depends on the specific mechanoreceptor(s) expressed by the cell. In this regard, a multitude of cell transmembrane proteins including various GPCRs[58, 91, 125], tyrosine kinase receptors[126-130], ion channels[131], NO synthases, and integrin-associated focal adhesions[132, 133] have been implicated as fluid shear stress transducers for a variety of cells (e.g., endothelial cells, osteoblasts, neutrophils) and microorganisms (e.g. dino-flagella)[134].

One potential action of fluid shear stress on transmembrane mechanosensors (e.g., FPR) is to alter their surface levels. In the case of GPCRs, exposing migrating neutrophil-like cells to parallel plate flow redistributes surface-associated FPRs to a perinuclear compartment in the cytosol[135]. These results suggest that internalization of FPRs under fluid shear stimulation leads to pseudopod retraction by counteracting their constitutive activity which drives pseudopod extension. It should be noted, however, that intact FPR must be present since cleavage of FPR is linked to an impaired ability of fluid shear stress to promote retraction of neutrophil pseudopods[136]. Since receptor internalization occurs across the lipid bilayer, shear-induced changes in mechanoreceptor surface levels may thus be a mechanosensitive neutrophil response influenced by properties of the cell membrane.

It is also feasible that the ability of shear stress to alter protein tertiary structure is a function of membrane properties. In addition to evidence regarding the influence of shear stress on the conformation of FPR and CD18 integrins, fluid flow also alters the structure of other membrane-bound GPCRs in other cell types including the bradykinin $\mathrm{B}_{2}$ receptor for endothelial cells and the type I parathyroid hormone receptor for osteoblasts[58, 125]. Interestingly, physiologically relevant magnitudes of mechanical stresses are capable of physically altering the conformation of proteins[132, 133, 137]. Since these proteins are embedded in the cell membrane, it is possible that membrane properties influence flowrelated perturbations of protein structure.

In the end, the physicochemical properties (e.g., fluidity, lipid rafts) of the cell membrane, with their influence on the ability of surface mechanosensors to adopt structural shifts under 
shear, come to the forefront in terms of how hypercholesterolemia modifies neutrophil mechanosensitivity. This is the topic of the next section.

\subsubsection{Membrane cholesterol versus membrane fluidity in hypercholesterolemic impairment} of neutrophil mechanosensitivity

Hypercholesterolemia is associated with chronic neutrophil activation and elevated blood cholesterol as well as cholesterol enrichment in the plasma membranes of blood cells. Based on the intimate relationship between protein dynamics (e.g., surface expression, conformational activity) and the cell membrane (as described in the previous section), the chemical and mechanical properties of the lipid bilayer may be critical determinants of the ability of neutrophils to sense fluid shear stress. Along this line, hypercholesterolemiarelated membrane perturbations may reduce the neutrophil responsiveness to shear stress by interfering with critical mechanotransduction events, e.g. GPCR and CD18 conformational activity, protease release, and/or production of ROS, that must bidirectionally transmit biological activity across the cell membrane (Figure 4).

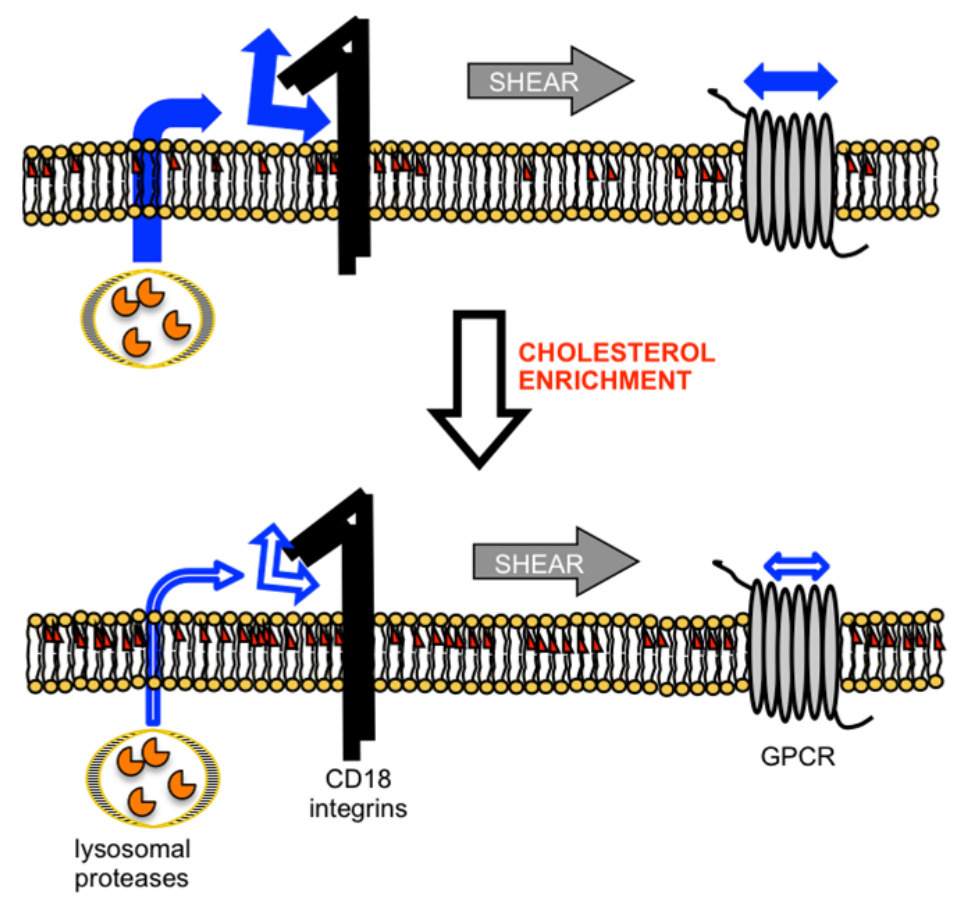

Figure 4. Effects of cholesterol abundance on neutrophil mechanotransduction. Elevations in extracellular cholesterol lead to membrane cholesterol enrichment which may alter cell mechanosensitivity either by influencing shear-induced structural changes of surface sensors, or by interfering with shear-induced release of lysosomal proteases. The cell membrane may also influence contributions from ROS/RNS (not shown). 


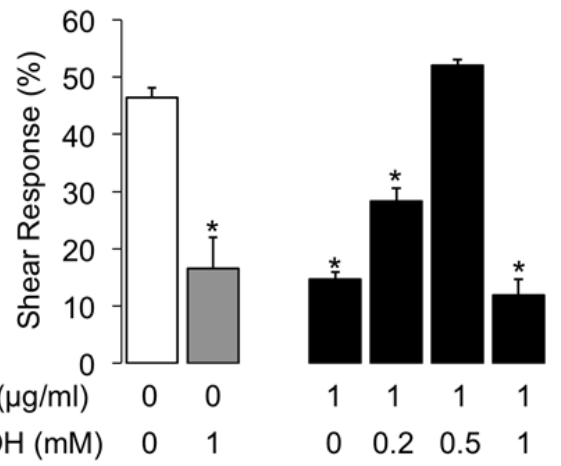

(A)

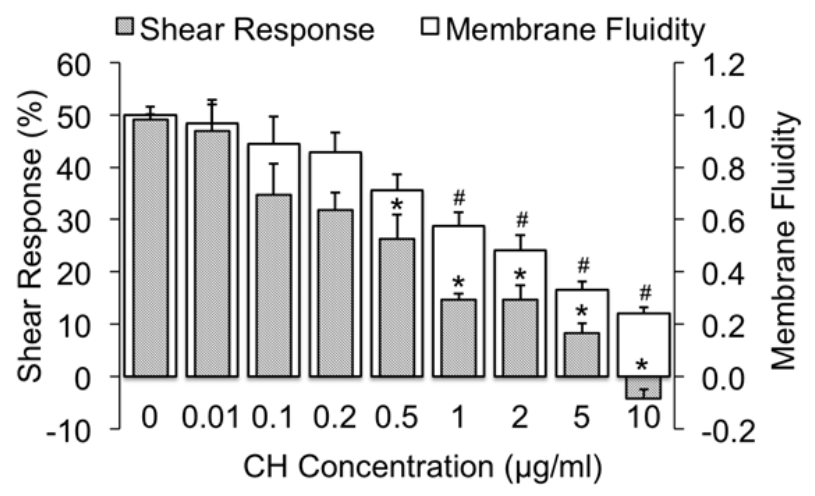

(B)

Figure 5. Relationship between membrane cholesterol-dependent fluidity and neutrophil shear responses. A: Recovery effects of benzyl alcohol ( $\mathrm{BnOH}$; a membrane fluidizer) on the shear response by neutrophils treated with cholesterol-enhancing agents $(\mathrm{CH})$. B: Does-dependent effects of cholesterol enrichment on neutrophil shear response and membrane fluidity. Cone-plate shear: $5 \mathrm{dyn} / \mathrm{cm}^{2}$ for 10 min. Bars are mean percentage of reductions in activated cells by shear \pm SEM. ${ }^{*}, \# \mathrm{p}<0.001$ compared to untreated cells using Student's t-test with Bonferrroni's adjustment.

Recently, we reported that neutrophil deactivation by shear stress depends on the cholesterol-dependent physicochemical properties (i.e., fluidity) of the cell membrane[40]. Fundamentally, we showed that the deactivating actions of fluid shear require a cell membrane containing an optimal level of cholesterol. Shear stress mechanotransduction is impaired if there is too much or too little cholesterol. Moreover, the membrane must be capable of supporting the formation of lipid rafts. But, the critical evidence from this work are our observations[40] that membrane fluidizer, benzyl alcohol, was capable of counteracting the rigidifying effects of membrane cholesterol enhancement (with 
cyclodextrin-cholesterol conjugates) and that the concentration of benzyl alcohol to achieve this depended on the amount of cholesterol loaded into the neutrophil membranes (Figure $5 \mathrm{~A})$. Thus, there is also an optimal membrane fluidity level permissive for shear-induced neutrophil deactivation. This was confirmed by regression analysis[40] which revealed a linear relationship (Figure 5B) between membrane cholesterol-related fluidity and the degree to which neutrophils within a population are inactivated by fluid flow. Membrane cholesterol enrichment therefore impairs neutrophil mechanosensitivity, at least in part, through its impact on membrane fluidity.

Interestingly, neutrophils from LDLr-/- mice fed a HFD exhibit a reduced and even reversed shear stress response relative to cells from similar mice maintained on a regular chow (i.e., normal) diet (ND)[40]. These observations were consistent with our in vitro data correlating membrane cholesterol levels with neutrophil mechanosensitivity[40]. In fact, the shear sensitivity of neutrophils from hypercholesterolemic mice tracks negatively with timedependent increases in blood levels of cholesterol, particularly of the free form (Figure 6). Presumably, the gradual loading of cholesterol into the neutrophil membrane resulting from the progressive increases in the cholesterol concentration gradient across the outer leaflet of the cell membrane is responsible for the time-dependent decrease in shear mechanosensitivity. Impairment of neutrophil shear responses by membrane cholesterol enrichment may thus underlie the pathogenesis of hypercholesterolemic disorders via an effect on cell membrane fluidity which governs the ability of protein sensors to initiate a sufficient degree of mechanotransduction at the cell surface. As such, a chronic inflammatory state may develop.

A LDLr-/- mice on ND

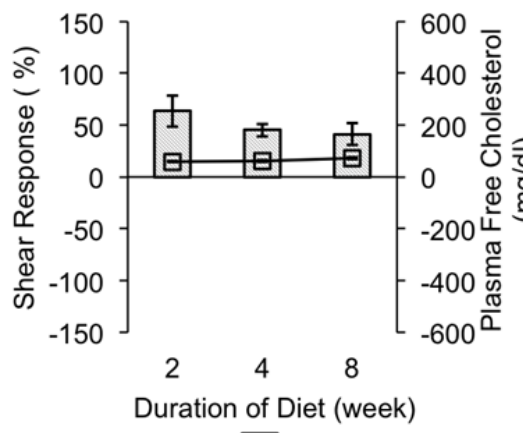

Shear Response
B LDLr-/- mice on HFD

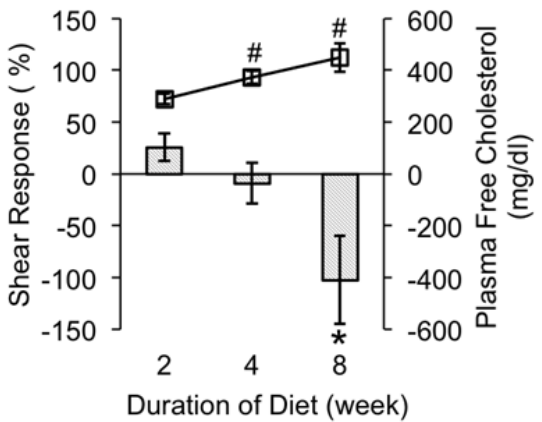

曰- Plasma Cholesterol

Figure 6. Correlation between neutrophil shear responses and serum levels of free cholesterol. A: LDLr-/- mice on normal diet (ND); B: LDLr-/- mice on high fat diet (HFD). Cone-plate shear: $5 \mathrm{dyn} / \mathrm{cm}^{2}$ for $10 \mathrm{~min}$. Bars and square dots are mean \pm SEM. ${ }^{*}, \# p<0.02$ compared to 2 -week using Student's t-test with Bonferrroni's adjustment. 


\section{Future directions}

To date, the accumulated evidence strongly points to shear stress mechanotransduction as an important negative control mechanism for neutrophils flowing in blood under noninflamed conditions and, thus, an important mediator of circulatory homeostasis. For the most part, the pathobiology of hypercholesterolemia is a process that takes decades to develop into a serious, life-threatening condition and tracks with gradual elevations in blood cholesterol levels. In addition, hypercholesterolemia is characterized by a chronic inflammatory phenotype associated with elevated levels of neutrophil activity in the blood. The question is how these two factors may be related or linked?

Based on the evidence presented in this chapter, the possibility that elevations in blood cholesterol levels impair the neutrophil-deactivating effects of fluid shear stress further suggests that vascular mechanotransduction is an important aspect of cardiovascular physiology and that the pathobiology of hypercholesterolemia may result, at least in part, from a putative disruption of this mechanotransducing function. This statement applies not only to neutrophils, but also to other cells in the circulation including the other white cells and the endothelium. Moreover, the presented evidence hints at the need to shift focus on the study of vascular mechanobiology from characterizing mechanotransduction (i.e., identifying mechanobiological signaling) in disease to actively investigating the influence of mechanosensitivity (i.e. the degree to which cells transduce fluid stresses) on vascular pathogenesis. In our case, we linked altered neutrophil mechanosensitivity with the gradual changes in blood cholesterol levels and leukocyte membranes during the development of hypercholesterolemia in LDLr-/- mice fed a fat-enriched diet. In light of our own evidence and those of others $[3,4,8]$ showing that shear stress is anti-inflammatory for neutrophils, it is possible that a putative source of vascular dysfunction causal for hypercholesterolemic pathobiology is the aberrant neutrophil mechanosensitivity.

Despite recognition that vascular mechanotransduction is critical for circulatory homeostasis, there are no markers currently in use or, to our knowledge, in development that account for mechanosensitivity to predict vascular inflammatory status. Current indicators of inflammation include C-reactive protein (CRP; $>3 \mathrm{mg} / \mathrm{L}$ is at cardiovascular risk) and serum amyloid protein $\mathrm{A}$ (SAA; $>10 \mathrm{mg} / \mathrm{L}$ is at cardiovascular risk). But even though these two biochemical markers are the gold standard measures of inflammatory activity for blood[138, 139], they are upregulated when leukocyte activity levels are already elevated. It is thus not clear whether these molecules are viable "predictors" or just indicators of chronic inflammatory disorders. As such, understanding, characterizing, and formulating measures of neutrophil mechanosensitivity may prove useful in revealing earlier clues regarding the state of inflammation in blood.

In the end, the likelihood that a cholesterol-dependent loss of neutrophil sensitivity to fluid flow stimuli leads to pathological situations implicates a wide range of cardiovascular (and non-cardiovascular) diseases that correlate with both chronic inflammation and an altered cholesterol environment, e.g. hypercholesterolemia and diabetes[74, 140]. The critical issues are to increase efforts to define the link between chronic inflammation and impaired 
neutrophil mechanotransduction and to determine if chronic inflammation precedes or results from an impairment of vascular mechanotransduction. Further work is, therefore, needed to determine mechanistic-level connections between the cell surface, the flow sensors, the extracellular flow environment, and the influence of a hypercholesterolemic environment on these. The hope is that by fully defining the role of fluid mechanics in the physiological regulation of leukocytes, particularly the neutrophils, one may gain a better understanding of their role in the pathogenesis of cardiovascular disease.

\section{Author details}

Xiaoyan Zhang and Hainsworth Y. Shin*

Center for Biomedical Engineering, University of Kentucky, Lexington, KY, USA

\section{References}

[1] Mazzoni, M.C. and G.W. Schmid-Schönbein, Mechanisms and consequences of cell activation in the microcirculation. Cardiovasc Res, 1996. 32(4): p. 709-19.

[2] Gorman C, P.K.D.A., and Cray D, The Fires Within. Time, 2004. 163: p. 30-46.

[3] Underwood, A., Quieting a Body's Defenses. Newsweek, 2005.

[4] Libby, P., P.M. Ridker, and A. Maseri, Inflammation and atherosclerosis. Circulation, 2002. 105(9): p. 1135-43.

[5] Steinberg, D., Atherogenesis in perspective: hypercholesterolemia and inflammation as partners in crime. Nat Med, 2002. 8(11): p. 1211-7.

[6] Boullier, A., et al., Scavenger receptors, oxidized LDL, and atherosclerosis. Ann N Y Acad Sci, 2001. 947: p. 214-22; discussion 222-3.

[7] Stokes, K.Y., et al., Hypercholesterolemia promotes inflammation and microvascular dysfunction: role of nitric oxide and superoxide. Free Radic Biol Med, 2002. 33(8): p. 1026-36.

[8] Scalia, R., J.Z. Appel, 3rd, and A.M. Lefer, Leukocyte-endothelium interaction during the early stages of hypercholesterolemia in the rabbit: role of P-selectin, ICAM-1, and VCAM-1. Arterioscler Thromb Vasc Biol, 1998. 18(7): p. 1093-100.

[9] Stokes, K.Y., et al., $N A D(P) H$ oxidase-derived superoxide mediates hypercholesterolemiainduced leukocyte-endothelial cell adhesion. Circ Res, 2001. 88(5): p. 499-505.

[10] Mori, N., et al., Ischemia-reperfusion induced microvascular responses in LDL-receptor -/mice. Am J Physiol, 1999. 276(5 Pt 2): p. H1647-54.

[11] Lehr, H.A., et al., P-selectin mediates the interaction of circulating leukocytes with platelets and microvascular endothelium in response to oxidized lipoprotein in vivo. Lab Invest, 1994. 71(3): p. 380-6.

[12] Vink, H., A.A. Constantinescu, and J.A. Spaan, Oxidized lipoproteins degrade the endothelial surface layer : implications for platelet-endothelial cell adhesion. Circulation, 2000. 101(13): p. 1500-2.

\footnotetext{
${ }^{*}$ Corresponding Author
} 
[13] Parthasarathy, S., D. Steinberg, and J.L. Witztum, The role of oxidized low-density lipoproteins in the pathogenesis of atherosclerosis. Annu Rev Med, 1992. 43: p. 219-25.

[14] Cunningham, K.S. and A.I. Gotlieb, The role of shear stress in the pathogenesis of atherosclerosis. Lab Invest, 2005. 85(1): p. 9-23.

[15] De Caterina, R., et al., Nitric oxide decreases cytokine-induced endothelial activation. Nitric oxide selectively reduces endothelial expression of adhesion molecules and proinflammatory cytokines. J Clin Invest, 1995. 96(1): p. 60-8.

[16] De Keulenaer, G.W., et al., Oscillatory and steady laminar shear stress differentially affect human endothelial redox state: role of a superoxide-producing NADH oxidase. Circ Res, 1998. 82(10): p. 1094-101.

[17] Nagel, T., et al., Shear stress selectively upregulates intercellular adhesion molecule-1 expression in cultured human vascular endothelial cells. J Clin Invest, 1994. 94(2): p. 885-91.

[18] Drechsler, M., et al., Hyperlipidemia-triggered neutrophilia promotes early atherosclerosis. Circulation, 2010. 122(18): p. 1837-45.

[19] Rotzius, P., et al., Distinct infiltration of neutrophils in lesion shoulders in ApoE-/- mice. Am J Pathol, 2010. 177(1): p. 493-500.

[20] van Leeuwen, M., et al., Accumulation of myeloperoxidase-positive neutrophils in atherosclerotic lesions in LDLR-/- mice. Arterioscler Thromb Vasc Biol, 2008. 28(1): p. 84-9.

[21] Ionita, M.G., et al., High neutrophil numbers in human carotid atherosclerotic plaques are associated with characteristics of rupture-prone lesions. Arterioscler Thromb Vasc Biol, 2010. 30(9): p. 1842-8.

[22] Naruko, T., et al., Neutrophil infiltration of culprit lesions in acute coronary syndromes. Circulation, 2002. 106(23): p. 2894-900.

[23] Davies, P.F., Hemodynamic shear stress and the endothelium in cardiovascular pathophysiology. Nat Clin Pract Cardiovasc Med, 2009. 6(1): p. 16-26.

[24] Burns, M.P. and N. DePaola, Flow-conditioned HUVECs support clustered leukocyte adhesion by coexpressing ICAM-1 and E-selectin. Am J Physiol Heart Circ Physiol, 2005. 288(1): p. H194-204.

[25] Maiellaro, K. and W.R. Taylor, The role of the adventitia in vascular inflammation. Cardiovasc Res, 2007. 75(4): p. 640-8.

[26] Mulligan-Kehoe, M.J., The vasa vasorum in diseased and nondiseased arteries. Am J Physiol Heart Circ Physiol, 2010. 298(2): p. H295-305.

[27] Ritman, E.L. and A. Lerman, The dynamic vasa vasorum. Cardiovasc Res, 2007. 75(4): p. 649-58.

[28] Soehnlein, O., Multiple roles for neutrophils in atherosclerosis. Circ Res, 2012. 110(6): p. 87588.

[29] Mazor, R., et al., Primed polymorphonuclear leukocytes constitute a possible link between inflammation and oxidative stress in hyperlipidemic patients. Atherosclerosis, 2008. 197(2): p. 937-43.

[30] Nicholls, S.J. and S.L. Hazen, Myeloperoxidase and cardiovascular disease. Arterioscler Thromb Vasc Biol, 2005. 25(6): p. 1102-11. 
[31] Chertov, O., et al., Identification of human neutrophil-derived cathepsin $G$ and azurocidin/CAP37 as chemoattractants for mononuclear cells and neutrophils. J Exp Med, 1997. 186(5): p. 739-47.

[32] Soehnlein, O., et al., Neutrophil secretion products pave the way for inflammatory monocytes. Blood, 2008. 112(4): p. 1461-71.

[33] Schmid-Schönbein, G.W., Analysis of inflammation. Annu Rev Biomed Eng, 2006. 8: p. 93131.

[34] Eppihimer, M.J. and H.H. Lipowsky, Effects of leukocyte-capillary plugging on the resistance to flow in the microvasculature of cremaster muscle for normal and activated leukocytes. Microvasc Res, 1996. 51(2): p. 187-201.

[35] Worthen, G.S., et al., Mechanics of stimulated neutrophils: cell stiffening induces retention in capillaries. Science, 1989. 245(4914): p. 183-6.

[36] Helmke, B.P., et al., A mechanism for erythrocyte-mediated elevation of apparent viscosity by leukocytes in vivo without adhesion to the endothelium. Biorheology, 1998. 35(6): p. 437-48.

[37] Lipowsky, H.H., Microvascular rheology and hemodynamics. Microcirculation, 2005. 12(1): p. 5-15.

[38] Lange, Y., et al., Plasma membranes contain half the phospholipid and 90\% of the cholesterol and sphingomyelin in cultured human fibroblasts. J Biol Chem, 1989. 264(7): p. 3786-93.

[39] Day, A.P., et al., Effect of simvastatin therapy on cell membrane cholesterol content and membrane function as assessed by polymorphonuclear cell NADPH oxidase activity. Ann Clin Biochem, 1997. 34 ( Pt 3): p. 269-75.

[40] Zhang, X., et al., Membrane cholesterol modulates the fluid shear stress response of polymorphonuclear leukocytes via its effects on membrane fluidity. Am J Physiol Cell Physiol, 2011. 301(2): p. C451-60.

[41] Oh, H., et al., Membrane cholesterol is a biomechanical regulator of neutrophil adhesion. Arterioscler Thromb Vasc Biol, 2009. 29(9): p. 1290-7.

[42] Cooper, R.A., Influence of increased membrane cholesterol on membrane fluidity and cell function in human red blood cells. J Supramol Struct, 1978. 8(4): p. 413-30.

[43] Goldstein, J.L. and M.S. Brown, The low-density lipoprotein pathway and its relation to atherosclerosis. Annu Rev Biochem, 1977. 46: p. 897-930.

[44] Brasaemle, D.L. and A.D. Attie, Rapid intracellular transport of LDL-derived cholesterol to the plasma membrane in cultured fibroblasts. J Lipid Res, 1990. 31(1): p. 103-12.

[45] Lara, L.L., et al., Low density lipoprotein receptor expression and function in human polymorphonuclear leucocytes. Clin Exp Immunol, 1997. 107(1): p. 205-12.

[46] Goldstein, J.L. and M.S. Brown, Binding and degradation of low density lipoproteins by cultured human fibroblasts. Comparison of cells from a normal subject and from a patient with homozygous familial hypercholesterolemia. J Biol Chem, 1974. 249(16): p. 5153-62.

[47] Basu, S.K., et al., Degradation of cationized low density lipoprotein and regulation of cholesterol metabolism in homozygous familial hypercholesterolemia fibroblasts. Proc Natl Acad Sci U S A, 1976. 73(9): p. 3178-82. 
[48] Phillips, M.C., W.J. Johnson, and G.H. Rothblat, Mechanisms and consequences of cellular cholesterol exchange and transfer. Biochim Biophys Acta, 1987. 906(2): p. 223-76.

[49] Brasaemle, D.L., A.D. Robertson, and A.D. Attie, Transbilayer movement of cholesterol in the human erythrocyte membrane. J Lipid Res, 1988. 29(4): p. 481-9.

[50] Ohvo-Rekila, H., et al., Cholesterol interactions with phospholipids in membranes. Prog Lipid Res, 2002. 41(1): p. 66-97.

[51] Rothman, J.E. and D.M. Engelman, Molecular mechanism for the interaction of phospholipid with cholesterol. Nat New Biol, 1972. 237(71): p. 42-4.

[52] Chabanel, A., et al., Influence of cholesterol content on red cell membrane viscoelasticity and fluidity. Biophys J, 1983. 44(2): p. 171-6.

[53] Coderch, L., et al., Influence of cholesterol on liposome fluidity by EPR. Relationship with percutaneous absorption. J Control Release, 2000. 68(1): p. 85-95.

[54] Lenaz, G., Lipid fluidity and membrane protein dynamics. Biosci Rep, 1987. 7(11): p. 823-37.

[55] Rimon, G., et al., Mode of coupling between hormone receptors and adenylate cyclase elucidated by modulation of membrane fluidity. Nature, 1978. 276(5686): p. 394-6.

[56] Schramm, M., Transfer of glucagon receptor from liver membranes to a foreign adenylate cyclase by a membrane fusion procedure. Proc Natl Acad Sci U S A, 1979. 76(3): p. 1174-8.

[57] Lenaz, G.a.P.C., G., Structure and Properties of Cell Membranes, G. Benga, Editor 1985, CRC Press: Boca Raton, FLA. p. 73-136.

[58] Chachisvilis, M., Y.L. Zhang, and J.A. Frangos, G protein-coupled receptors sense fluid shear stress in endothelial cells. Proc Natl Acad Sci U S A, 2006. 103(42): p. 15463-8.

[59] Simons, K. and D. Toomre, Lipid rafts and signal transduction. Nat Rev Mol Cell Biol, 2000. 1(1): p. 31-9.

[60] Brown, D.A. and E. London, Functions of lipid rafts in biological membranes. Annu Rev Cell Dev Biol, 1998. 14: p. 111-36.

[61] Radel, C., M. Carlile-Klusacek, and V. Rizzo, Participation of caveolae in beta1 integrinmediated mechanotransduction. Biochem Biophys Res Commun, 2007. 358(2): p. 626-31.

[62] Rizzo, V., et al., In situ flow activates endothelial nitric oxide synthase in luminal caveolae of endothelium with rapid caveolin dissociation and calmodulin association. J Biol Chem, 1998. 273(52): p. 34724-9.

[63] Rizzo, V., et al., Rapid mechanotransduction in situ at the luminal cell surface of vascular endothelium and its caveolae. J Biol Chem, 1998. 273(41): p. 26323-9.

[64] Marwali, M.R., et al., Membrane cholesterol regulates LFA-1 function and lipid raft heterogeneity. Blood, 2003. 102(1): p. 215-22.

[65] Niggli, V., et al., Impact of cholesterol depletion on shape changes, actin reorganization, and signal transduction in neutrophil-like HL-60 cells. Exp Cell Res, 2004. 296(2): p. 358-68.

[66] Pierini, L.M., et al., Membrane lipid organization is critical for human neutrophil polarization. J Biol Chem, 2003. 278(12): p. 10831-41.

[67] Seely, A.J., J.L. Pascual, and N.V. Christou, Science review: Cell membrane expression (connectivity) regulates neutrophil delivery, function and clearance. Crit Care, 2003. 7(4): p. 291-307. 
[68] Tuluc, F., J. Meshki, and S.P. Kunapuli, Membrane lipid microdomains differentially regulate intracellular signaling events in human neutrophils. Int Immunopharmacol, 2003. 3(13-14): p. $1775-90$.

[69] Stulc, T., et al., Leukocyte and endothelial adhesion molecules in patients with hypercholesterolemia: the effect of atorvastatin treatment. Physiol Res, 2008. 57(2): p. 184-94.

[70] Lehr, H.A., et al., In vitro effects of oxidized low density lipoprotein on CD11b/CD18 and Lselectin presentation on neutrophils and monocytes with relevance for the in vivo situation. Am J Pathol, 1995. 146(1): p. 218-27.

[71] Sugano, R., et al., Polymorphonuclear leukocytes may impair endothelial function: results of crossover randomized study of lipid-lowering therapies. Arterioscler Thromb Vasc Biol, 2005. 25(6): p. 1262-7.

[72] Furlow, M. and S.L. Diamond, Interplay between membrane cholesterol and ethanol differentially regulates neutrophil tether mechanics and rolling dynamics. Biorheology, 2011. 48(1): p. 49-64.

[73] Tailor, A. and D.N. Granger, Hypercholesterolemia promotes leukocyte-dependent platelet adhesion in murine postcapillary venules. Microcirculation, 2004. 11(7): p. 597-603.

[74] Lechi, C., et al., Increased leukocyte aggregation in patients with hypercholesterolaemia. Clin Chim Acta, 1984. 144(1): p. 11-6.

[75] Araujo, F.B., et al., Evaluation of oxidative stress in patients with hyperlipidemia. Atherosclerosis, 1995. 117(1): p. 61-71.

[76] Kristal, B., et al., Participation of peripheral polymorphonuclear leukocytes in the oxidative stress and inflammation in patients with essential hypertension. Am J Hypertens, 1998. 11(8 Pt 1): p. 921-8.

[77] Shurtz-Swirski, R., et al., Involvement of peripheral polymorphonuclear leukocytes in oxidative stress and inflammation in type 2 diabetic patients. Diabetes Care, 2001. 24(1): p. 104-10.

[78] Makino, A., et al., Mechanotransduction in leukocyte activation: a review. Biorheology, 2007. 44(4): p. 221-49.

[79] Shin, H.Y., et al., Mechanobiological Evidence for the Control of Neutrophil Activity by Fluid Shear Stress in Mechanobiology Handbook, J. Nagatomi, Editor 2011, CRC Press: Boca Raton, FL, USA. p. 139-75.

[80] Fukuda, S. and G.W. Schmid-Schönbein, Centrifugation attenuates the fluid shear response of circulating leukocytes. J Leukoc Biol, 2002. 72(1): p. 133-9.

[81] Fukuda, S., et al., Mechanisms for regulation of fluid shear stress response in circulating leukocytes. Circ Res, 2000. 86(1): p. E13-8.

[82] Moazzam, F., et al., The leukocyte response to fluid stress. Proc Natl Acad Sci U S A, 1997. 94(10): p. 5338-43.

[83] Makino, A., et al., Control of neutrophil pseudopods by fluid shear: role of Rho family GTPases. Am J Physiol Cell Physiol, 2005. 288(4): p. C863-71.

[84] Komai, Y. and G.W. Schmid-Schönbein, De-activation of neutrophils in suspension by fluid shear stress: a requirement for erythrocytes. Ann Biomed Eng, 2005. 33(10): p. 1375-86. 
[85] Shive, M.S., W.G. Brodbeck, and J.M. Anderson, Activation of caspase 3 during shear stressinduced neutrophil apoptosis on biomaterials. J Biomed Mater Res, 2002. 62(2): p. 163-8.

[86] Shive, M.S., M.L. Salloum, and J.M. Anderson, Shear stress-induced apoptosis of adherent neutrophils: a mechanism for persistence of cardiovascular device infections. Proc Natl Acad Sci U S A, 2000. 97(12): p. 6710-5.

[87] Chen, H.Q., et al., Effect of steady and oscillatory shear stress on F-actin content and distribution in neutrophils. Biorheology, 2004. 41(5): p. 655-64.

[88] Cicchetti, G., P.G. Allen, and M. Glogauer, Chemotactic signaling pathways in neutrophils: from receptor to actin assembly. Crit Rev Oral Biol Med, 2002. 13(3): p. 220-8.

[89] Niggli, V., Signaling to migration in neutrophils: importance of localized pathways. Int J Biochem Cell Biol, 2003. 35(12): p. 1619-38.

[90] Tybulewicz, V.L. and R.B. Henderson, Rho family GTPases and their regulators in lymphocytes. Nat Rev Immunol, 2009. 9(9): p. 630-44.

[91] Makino, A., et al., G protein-coupled receptors serve as mechanosensors for fluid shear stress in neutrophils. Am J Physiol Cell Physiol, 2006. 290(6): p. C1633-9.

[92] Marschel, P. and G.W. Schmid-Schönbein, Control of fluid shear response in circulating leukocytes by integrins. Ann Biomed Eng, 2002. 30(3): p. 333-43.

[93] Anderson, S.I., et al., Linked regulation of motility and integrin function in activated migrating neutrophils revealed by interference in remodelling of the cytoskeleton. Cell Motil Cytoskeleton, 2003. 54(2): p. 135-46.

[94] Simon, S.I. and C.E. Green, Molecular mechanics and dynamics of leukocyte recruitment during inflammation. Annu Rev Biomed Eng, 2005. 7: p. 151-85.

[95] Simon, S.I. and H.L. Goldsmith, Leukocyte adhesion dynamics in shear flow. Ann Biomed Eng, 2002. 30(3): p. 315-32.

[96] Shin, H.Y., S.I. Simon, and G.W. Schmid-Schönbein, Fluid shear-induced activation and cleavage of CD18 during pseudopod retraction by human neutrophils. J Cell Physiol, 2008. 214(2): p. 528-36.

[97] Fukuda, S. and G.W. Schmid-Schönbein, Regulation of CD18 expression on neutrophils in response to fluid shear stress. Proc Natl Acad Sci U S A, 2003. 100(23): p. 13152-7.

[98] Walcheck, B., et al., Neutrophil rolling altered by inhibition of L-selectin shedding in vitro. Nature, 1996. 380(6576): p. 720-3.

[99] Carney, D.F., et al., Effect of serine proteinase inhibitors on neutrophil function: alpha-1proteinase inhibitor, antichymotrypsin, and a recombinant hybrid mutant of antichymotrypsin (LEX032) modulate neutrophil adhesion interactions. J Leukoc Biol, 1998. 63(1): p. 75-82.

[100] Pfaff, M., X. Du, and M.H. Ginsberg, Calpain cleavage of integrin beta cytoplasmic domains. FEBS Lett, 1999. 460(1): p. 17-22.

[101] Arnaout, M.A., Structure and function of the leukocyte adhesion molecules CD11/CD18. Blood, 1990. 75(5): p. 1037-50.

[102] Radi, Z.A., M.E. Kehrli, Jr., and M.R. Ackermann, Cell adhesion molecules, leukocyte trafficking, and strategies to reduce leukocyte infiltration. J Vet Intern Med, 2001. 15(6): p. 516-29. 
[103] Fialkow, L., Y. Wang, and G.P. Downey, Reactive oxygen and nitrogen species as signaling molecules regulating neutrophil function. Free Radic Biol Med, 2007. 42(2): p. 153-64.

[104] Guzik, T.J., R. Korbut, and T. Adamek-Guzik, Nitric oxide and superoxide in inflammation and immune regulation. J Physiol Pharmacol, 2003. 54(4): p. 469-87.

[105] Dal Secco, D., et al., Nitric oxide inhibits neutrophil migration by a mechanism dependent on ICAM-1: role of soluble guanylate cyclase. Nitric Oxide, 2006. 15(1): p. 77-86.

[106] Kubes, P., M. Suzuki, and D.N. Granger, Nitric oxide: an endogenous modulator of leukocyte adhesion. Proc Natl Acad Sci U S A, 1991. 88(11): p. 4651-5.

[107] Kubes, P., et al., Nitric oxide synthesis inhibition induces leukocyte adhesion via superoxide and mast cells. Faseb J, 1993. 7(13): p. 1293-9.

[108] Gaboury, J., et al., Nitric oxide prevents leukocyte adherence: role of superoxide. Am J Physiol, 1993. 265(3 Pt 2): p. H862-7.

[109] Vilhardt, F. and B. van Deurs, The phagocyte NADPH oxidase depends on cholesterolenriched membrane microdomains for assembly. EMBO J, 2004. 23(4): p. 739-48.

[110] Fukuda, S., et al., Contribution of fluid shear response in leukocytes to hemodynamic resistance in the spontaneously hypertensive rat. Circ Res, 2004. 95(1): p. 100-8.

[111] Suzuki, H., et al., Impaired leukocyte-endothelial cell interaction in spontaneously hypertensive rats. Hypertension, 1994. 24(6): p. 719-27.

[112] Arndt, H., C.W. Smith, and D.N. Granger, Leukocyte-endothelial cell adhesion in spontaneously hypertensive and normotensive rats. Hypertension, 1993. 21(5): p. 667-73.

[113] Suematsu, M., et al., The inflammatory aspect of the microcirculation in hypertension: oxidative stress, leukocytes/endothelial interaction, apoptosis. Microcirculation, 2002. 9(4): p. 259-76.

[114] Kaul, S., R.C. Padgett, and D.D. Heistad, Role of platelets and leukocytes in modulation of vascular tone. Ann N Y Acad Sci, 1994. 714: p. 122-35.

[115] Mugge, A., et al., Activation of leukocytes with complement C5a is associated with prostanoid-dependent constriction of large arteries in atherosclerotic monkeys in vivo. Atherosclerosis, 1992. 95(2-3): p. 211-22.

[116] Faraci, F.M., et al., Effect of atherosclerosis on cerebral vascular responses to activation of leukocytes and platelets in monkeys. Stroke, 1991. 22(6): p. 790-6.

[117] DeLano, F.A. and G.W. Schmid-Schönbein, Enhancement of glucocorticoid and mineralocorticoid receptor density in the microcirculation of the spontaneously hypertensive rat. Microcirculation, 2004. 11(1): p. 69-78.

[118] Sutanto, W., et al., Corticosteroid receptor plasticity in the central nervous system of various rat models. Endocr Regul, 1992. 26(3): p. 111-8.

[119] Fukuda, S., H. Mitsuoka, and G.W. Schmid-Schönbein, Leukocyte fluid shear response in the presence of glucocorticoid. J Leukoc Biol, 2004. 75(4): p. 664-70.

[120] Sugihara-Seki, M. and G.W. Schmid-Schönbein, The fluid shear stress distribution on the membrane of leukocytes in the microcirculation. J Biomech Eng, 2003. 125(5): p. 628-38. 
[121] Haidekker, M.A., N. L'Heureux, and J.A. Frangos, Fluid shear stress increases membrane fluidity in endothelial cells: a study with DCVJ fluorescence. Am J Physiol Heart Circ Physiol, 2000. 278(4): p. H1401-6.

[122] Butler, P.J., et al., Rate sensitivity of shear-induced changes in the lateral diffusion of endothelial cell membrane lipids: a role for membrane perturbation in shear-induced MAPK activation. Faseb J, 2002. 16(2): p. 216-8.

[123] Butler, P.J., et al., Shear stress induces a time- and position-dependent increase in endothelial cell membrane fluidity. Am J Physiol Cell Physiol, 2001. 280(4): p. C962-9.

[124] Ferraro, J.T., et al., Depletion of plasma membrane cholesterol dampens hydrostatic pressure and shear stress-induced mechanotransduction pathways in osteoblast cultures. Am J Physiol Cell Physiol, 2004. 286(4): p. C831-9.

[125] Zhang, Y.L., J.A. Frangos, and M. Chachisvilis, Mechanical stimulus alters conformation of type 1 parathyroid hormone receptor in bone cells. Am J Physiol Cell Physiol, 2009. 296(6): p. C1391-9.

[126] Chen, K.D., et al., Mechanotransduction in response to shear stress. Roles of receptor tyrosine kinases, integrins, and Shc. J Biol Chem, 1999. 274(26): p. 18393-400.

[127] Jin, Z.G., et al., Ligand-independent activation of vascular endothelial growth factor receptor 2 by fluid shear stress regulates activation of endothelial nitric oxide synthase. Circ Res, 2003. 93(4): p. 354-63.

[128] Lee, H.J. and G.Y. Koh, Shear stress activates Tie2 receptor tyrosine kinase in human endothelial cells. Biochem Biophys Res Commun, 2003. 304(2): p. 399-404.

[129] Milkiewicz, M., et al., HIF-1alpha and HIF-2alpha play a central role in stretch-induced but not shear-stress-induced angiogenesis in rat skeletal muscle. J Physiol, 2007. 583(Pt 2): p. 753-66.

[130] Shay-Salit, A., et al., VEGF receptor 2 and the adherens junction as a mechanical transducer in vascular endothelial cells. Proc Natl Acad Sci U S A, 2002. 99(14): p. 9462-7.

[131] Tarbell, J.M., S. Weinbaum, and R.D. Kamm, Cellular fluid mechanics and mechanotransduction. Ann Biomed Eng, 2005. 33(12): p. 1719-23.

[132] Kamm, R.D. and M.R. Kaazempur-Mofrad, On the molecular basis for mechanotransduction. Mech Chem Biosyst, 2004. 1(3): p. 201-9.

[133] Lee, S.E., R.D. Kamm, and M.R. Mofrad, Force-induced activation of talin and its possible role in focal adhesion mechanotransduction. J Biomech, 2007. 40(9): p. 2096-106.

[134] Chen, A.K., et al., Evidence for the role of G-proteins in flow stimulation of dinoflagellate bioluminescence. Am J Physiol Regul Integr Comp Physiol, 2007. 292(5): p. R2020-7.

[135] Su, S.S. and G.W. Schmid-Schönbein, Internalization of Formyl Peptide Receptor in Leukocytes Subject to Fluid Stresses. Cell Mol Bioeng, 2010. 3(1): p. 20-29.

[136] Chen, A.Y., et al., Receptor cleavage reduces the fluid shear response in neutrophils of the spontaneously hypertensive rat. Am J Physiol Cell Physiol, 2010. 299(6): p. C1441-9.

[137] Mofrad, M.R., et al., Force-induced unfolding of the focal adhesion targeting domain and the influence of paxillin binding. Mech Chem Biosyst, 2004. 1(4): p. 253-65.

[138] Gillmore, J.D., et al., Amyloid load and clinical outcome in AA amyloidosis in relation to circulating concentration of serum amyloid A protein. Lancet, 2001. 358(9275): p. 24-9. 
[139] Johnson, B.D., et al., Serum amyloid $A$ as a predictor of coronary artery disease and cardiovascular outcome in women: the National Heart, Lung, and Blood Institute-Sponsored Women's Ischemia Syndrome Evaluation (WISE). Circulation, 2004. 109(6): p. 726-32.

[140] Tomida, K., et al., Hypercholesterolemia induces leukocyte entrapment in the retinal microcirculation of rats. Curr Eye Res, 2001. 23(1): p. 38-43. 